\title{
Fiber Reinforced Calcium Phosphate Cement
}

\author{
*Luís Alberto dos Santos, †Luci Cristina de Oliveira, †Eliana Cristina da Silva Rigo, \\ $\$$ Raúl Garcia Carrodéguas; †Anselmo Ortega Boschi, and \\ *Antônio Celso Fonseca de Arruda

\begin{abstract}
*Faculdade de Engenharia Mecânica, Universidade Estadual de Campinas, Campinas; †Departamento de Engenharia de Materiais, Universidade Federal de São Carlos, São Paulo, Brazil; and \$Centro de Biomateriales, Universidad de La Habana, Havana, Cuba
\end{abstract}

\begin{abstract}
The term calcium phosphate cement was introduced by Gruninger et al. (1). This type of cement can be prepared by reacting a calcium phosphate salt with an aqueous solution, which causes it to set by the crossing of the precipitated crystals. These cements offer a series of advantages that allow their use as grafts and substitutes of damaged parts of the bone system. However, these cements have low mechanical strength compared to human bones. This work studied the influence of the use of polyamide fibers in the mechanical properties of a calcium
\end{abstract}

phosphate cement based on $\alpha$-tricalcium phosphate as well as the mechanisms involved in the increase of mechanical strength. The results demonstrate the feasibility of the use of polymeric fibers to increase mechanical strength and the need for coupling agents for the effective performance of the fibers as reinforcement in these materials. Key Words: Calcium phosphate cement-Calcium phosphate- $\alpha$-tricalcium phosphate-Bioceramic-Biomaterial-Medical devices.
The term calcium phosphate cement was introduced by Gruninger et al. (1), who stated that this type of cement can be prepared by mixing a calcium phosphate salt with water or with an aqueous solution to form a paste that can react at ambient or body temperatures to produce a precipitate containing one or more calcium phosphates, set by the intercrossing of precipitated crystals. They are constituted of one basic component and one acid component that, when mixed with water, react to form one or several products having a medium acidity.

These cements offer advantages that permit their use as grafts and substitutes of the damaged parts of the bone system, including moldability, minimum bone cavity, complete adaptation to the bone cavity, preparation during surgery, direct in vivo insertion,

\footnotetext{
Received August 1999.

Presented in part at the 1st Latin American Congress for Artificial Organs and Biomaterials-COLAOB-98, held in Belo Horizonte, Brazil, December 10-13, 1998.

Address correspondence and reprint requests to Dr. Luís Alberto dos Santos, Faculdade de Engenharia Mecânica, Universidade Estadual de Campinas, Caixa Postal 6136, CEP 13081-970, Campinas, SP, Brazil. E-mail: p-las@power.ufscar.br
}

setting in situ, optimal bone implant contact, and biocompatibility and bioativity.

Moreover, calcium phosphate cements can be used as drug delivery systems to which several medications or drugs such as antibiotic, antitumor, antiinflammatory drugs (2), and so on can easily be added. The main disadvantage of calcium phosphate cements is their low mechanical resistance which, in the best of cases, is equal to the trabecular bone, or to $20 \%$ of the cortical bone. The literature contains no in-depth studies on increasing the mechanical resistance of these cements. The availability of calcium phosphate cements with mechanical resistance close to human bones would considerably broaden the field of potential applications, which is currently limited to dentistry and maxillofacial surgery. Stronger compositions of calcium phosphate cements would be applicable in orthopedics and neurosurgery as an alternative for the knitting of multiple fractures of long bones, fixing of cemented articulation prostheses, iliac crest prostheses, intervertebral spacers, and substitution of vertebral bodies.

The objective of this work was to study the influence of the use of polyamide fibers in the mechanical resistance of a calcium phosphate cement based on 
$\alpha$-tricalcium phosphate as well as the mechanisms involved in the increase of mechanical resistance.

\section{MATERIALS AND METHODS}

The $\alpha$-tricalcium phosphate $(\alpha$-TCP) was prepared as described in the literature (3) by the reaction:

$$
\mathrm{CaCO}_{3}+\gamma-\mathrm{Ca}_{2} \mathrm{P}_{2} \mathrm{O}_{7} \rightarrow \alpha-\mathrm{Ca}_{3}\left(\mathrm{PO}_{4}\right)_{2}
$$

The reagents were mixed in a zirconia ball mill and calcinated at $1,300^{\circ} \mathrm{C}$ for $15 \mathrm{~h}$, followed by quenching on a stainless steel plate (4). The reaction product was ground in a polyethylene jar with zirconia balls in an alcoholic medium (anhydrous ethanol). The distribution of particle sizes was determined by granulometric distribution by sedimentation, using a Sedigraph-Micromeritics 5100 machine, with isopropanol as the dispersing agent. Organic monomers monofunctional acrylamide (5\%), and difunctional $\mathrm{N}, \mathrm{N}$ '-methylenebisacrylamide $(0.5 \%)$ were added to the $\alpha$-TCP, and the reaction was accelerated by the $\mathrm{N}, \mathrm{N}, \mathrm{N}^{\prime}, \mathrm{N}^{\prime}$-tetramethylethylenediamide $(0.25 \%)$ catalyst, with ammonium persulfate $(0.1 \%)$ used as the initiator. A 2.5\% $\mathrm{NaHPO}_{4}$ solution was also used to improve the $\alpha$-TCP cement setting reaction, and precipitated hydroxyapatite $(2 \%)$ was used as seed grains. The liquid-to-powder ratio was $0.55 \mathrm{ml} / \mathrm{g}$. (Nylon 66) with $0.1 \mathrm{~mm}$ diameter and $3 \mathrm{~mm}$ height in the following percentages (wt\%): 0.0, 0.2, 0.4, 0.8, and 1.6 was added to this polyamide fiber composition. The compressive strength was measured in a Universal Testing Machine (Model TTDML, Instron Corporation, Canton, MA, U.S.A.) at a loading rate of $1 \mathrm{~mm} / \mathrm{min}$ with the samples covered with wet filter paper. The $\alpha$-TCP powder and the cement with no fibers were analyzed by qualitative $\mathrm{x}$-ray diffraction, using a Model D 5000 x-ray diffratometer (Siemens, Karlshüre) with $\mathrm{CuK}_{\alpha}$ radiation and Ni filter, generated at $40 \mathrm{kV}$ and $40 \mathrm{~mA}$. The angular interval ( $2 \theta$ analyzed was from 20 to $40^{\circ}$, with goniometer speed at $2 \% \mathrm{~min}$. Five samples measuring $6 \mathrm{~mm}$ in diameter and $12 \mathrm{~mm}$ in height were prepared from each composition using a silicon mold. The samples were maintained at $100 \%$ relative humidity for $24 \mathrm{~h}$, and then placed in polystyrene flasks with Simulated Body Fluid (SBF) for 10 days at $37 \pm 1{ }^{\circ} \mathrm{C}$ (liquid/ solid volumetric ratio $=50$ ). The compressive measurements were taken after this 10 day period.

Some pieces taken after the compressive measurement were covered with gold and examined under scanning electron microscopy (Carl-Zeiss I Model DSM 940A) to determine their porosity and density using the Archimedes method in water.

\section{RESULTS AND DISCUSSION}

The $\alpha$-TCP obtained as described earlier produced a composition containing $\alpha$-TCP impurity and hydroxyapatite (HA) (5\% and 2\%, respectively), determined by quantitative $x$-ray diffraction. The x-ray spectra is presented in Fig. 1.

The presence of $\beta$-TCP as an impurity in the $\alpha$-TCP has been reported by several authors (5-8). There are no published reports of pure $\alpha$-TCP obtained and, normally, the $\alpha$-TCP obtained contains about $15 \%$ of $\beta$-TCP (9). Moreover, HA was detected as an impurity in the $\beta$-TCP. The presence of the HA can be explained by the balance reaction: $\mathrm{HA} \leftrightarrow \beta-\mathrm{TCP} \leftrightarrow \alpha-\mathrm{TCP}$, in which the speed of the first reverse reaction is smaller than the direct reaction (6).

Grain size distribution obtained by the sedimentation method showed a distribution of particle sizes ranging from 15 to $2 \mu \mathrm{m}$ with an average diameter of $7.5 \mu \mathrm{m}$ (this size being finer than that reported in the literature), which had a distribution between 20 and $2 \mu \mathrm{m}$. This explains the larger amount of liquid used containing $2.5 \%$ of $\mathrm{Na}_{2} \mathrm{HPO}_{4}(0.55 \mathrm{ml} / \mathrm{g})$ than that reported in the literature $(0.32 \mathrm{ml} / \mathrm{g})(10,11)$ because particle size distribution of powder in a cement is a decisive factor for the liquid/powder ratio needed to obtain a paste with an adequate consistency. Figure 2 shows the $\alpha$-TCP cement $\mathrm{x}$-ray spectra with no fibers after immersion in SBF for 8 days.

An increased intensity of the diffraction lines is observed for HA, with the decreased intensity if diffraction lines corresponding to the $\alpha$-TCP, and the almost complete disappearance of the $\beta$-TCP diffraction lines, which demonstrates the formation of

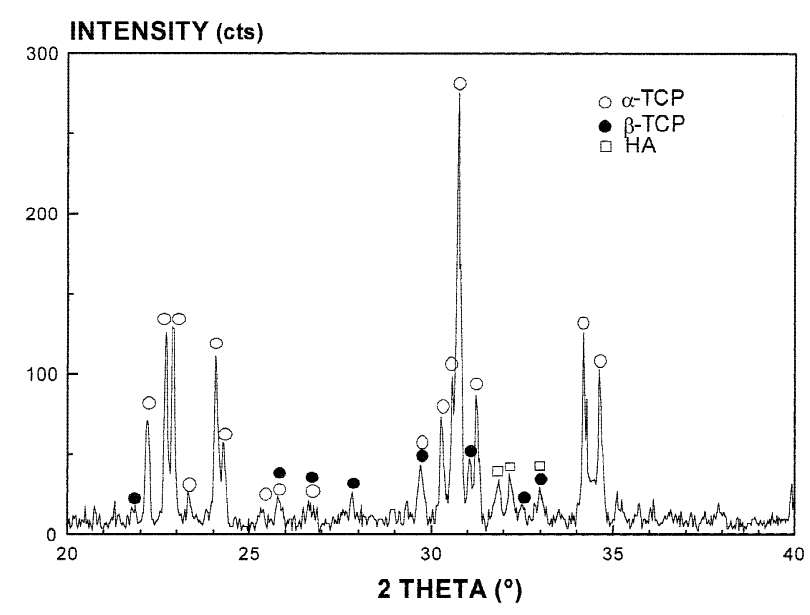

FIG. 1. The graph shows the $\alpha$-TCP $x$-ray spectra obtained from the $\gamma-\mathrm{Ca}_{2} \mathrm{P}_{2} \mathrm{O}_{7}$ and $\mathrm{CaCO}_{3}$ reaction at $1,300^{\circ} \mathrm{C}$ for $15 \mathrm{~h}$, followed by quenching. 


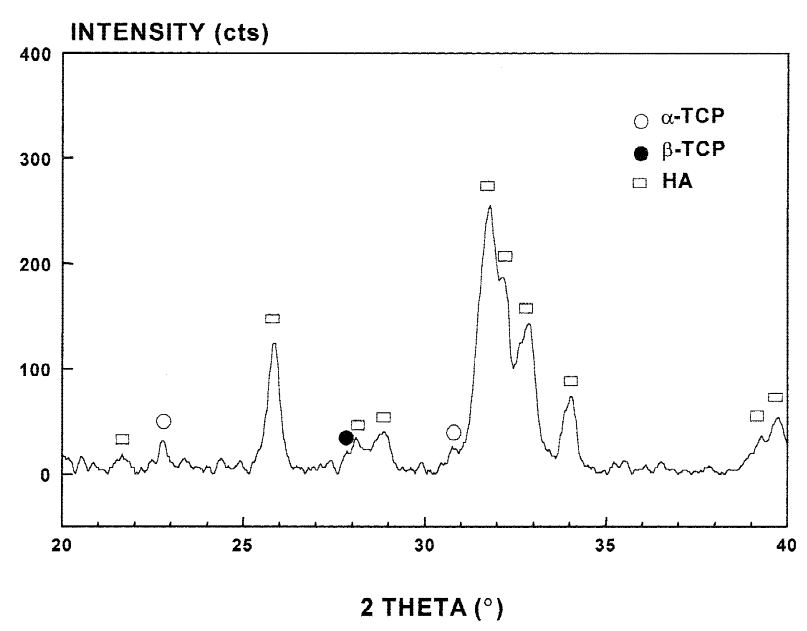

FIG. 2. The graph shows the $x$-ray spectra of cement after 8 days of immersion in SBF.

quite a high amount of HA. This was not, however, evaluated in the present study.

Porosity, density, and water absorption values, considering the standard deviation values, are similar for all compositions except in the case of the composition with no fiber addition, which presented a high value of porosity and water absorption with a resulting decrease of density and mechanical strength (Table 1). All compression strength values are lower than those obtained by Driessens et al. (10). However, the amount of liquid used by these authors $(0.32 \mathrm{ml} / \mathrm{g})$ was less than that used in this study $(0.55 \mathrm{ml} / \mathrm{g})$, which may explain the reduced values of the mechanical properties.

The addition of fibers to the cement composition produced a slight increase of compression strength, which was more accentuated in the compositions containing 0.2 and $0.8 \%$ of fibers (about $30 \%$ ). This represents extremely low increases when compared with the increases produced by polymeric fibers in Portland cement, which range from approximately 100 to $700 \%$ (12). In the case of additions of 0.4 and $1.6 \%$, there was a decrease in compression strength compared with the values obtained for the composi-

TABLE 1. Cements with fiber additions after 10 days in $S B F$

\begin{tabular}{ccccc}
\hline $\begin{array}{c}\text { Fiber } \\
\%\end{array}$ & $\begin{array}{c}\sigma_{\mathrm{c}} \\
(\mathrm{MPa})\end{array}$ & $\begin{array}{c}\mathrm{P} \\
(\%)\end{array}$ & $\begin{array}{c}\mathrm{D} \\
\left(\mathrm{g} / \mathrm{cm}^{3}\right)\end{array}$ & $\begin{array}{c}\mathrm{A} \\
(\%)\end{array}$ \\
\hline 0.0 & $9.5 \pm 0.5$ & $55.1 \pm 0.3$ & $1.26 \pm 0.01$ & $43.6 \pm 0.7$ \\
0.2 & $12.5 \pm 0.8$ & $53.9 \pm 0.4$ & $1.29 \pm 0.01$ & $41.9 \pm 0.4$ \\
0.4 & $9.5 \pm 0.8$ & $53.8 \pm 0.4$ & $1.29 \pm 0.01$ & $41.7 \pm 0.7$ \\
0.8 & $13.0 \pm 0.7$ & $52.4 \pm 0.4$ & $1.30 \pm 0.01$ & $40.2 \pm 0.5$ \\
1.6 & $9.8 \pm 0.7$ & $53.5 \pm 0.4$ & $1.28 \pm 0.02$ & $41.7 \pm 0.8$ \\
\hline
\end{tabular}

$\sigma_{\mathrm{c}}$ : compression strength, P: porosity, D: density, A: water absorption. tions containing 0.2 and $0.8 \%$. These values are very close to the values of the fiberless composition, which suggests a nonhomogeneous distribution possibly due to the reduced size of the samples. This may have caused the presence of fiberless zones with little mechanical resistance, which would cause flaws in the material due to the propagation of cracks through these areas, resulting in values similar to those of the fiberless composition. Moreover, the variation in mechanical resistance of several compositions does not allow a correlation to be made with variations in porosity, density, and water absorption because they are similar values. No observation of the reduction in mechanical resistance for all amounts of added fibers is indicative of fiber coupling because the addition of fibers that do not couple to the matrix could work as macro defects resulting in a strong reduction of mechanical resistance. This effect was not observed. Figures 3 and 4 show the microstructure of the compositions with and without the addition of polymeric fibers.

The morphology depicted in Fig. 3 is similar to that obtained in biomimetic procedures for $\mathrm{BaTiO}_{3}$ (13) coatings through immersion of the titanate in SBF after treatment in a solution containing $G$ glass or sodium silicate and is also similar to the format obtained from HA precipitation through the aqueous reaction of $\mathrm{CaOH}$ and $\mathrm{H}_{3} \mathrm{PO}_{4}$ (14). This, added to the $\mathrm{x}$-ray diffraction patterns (Fig. 2) which present diffraction lines corresponding only to the HA phase, leads us to assume that HA has precipitated into a spheroidal format.

Figure $4 \mathrm{~A}$ shows a polyamide fiber on the fractured surface of the cement, showing a space between the fiber and the cement. It also can be seen

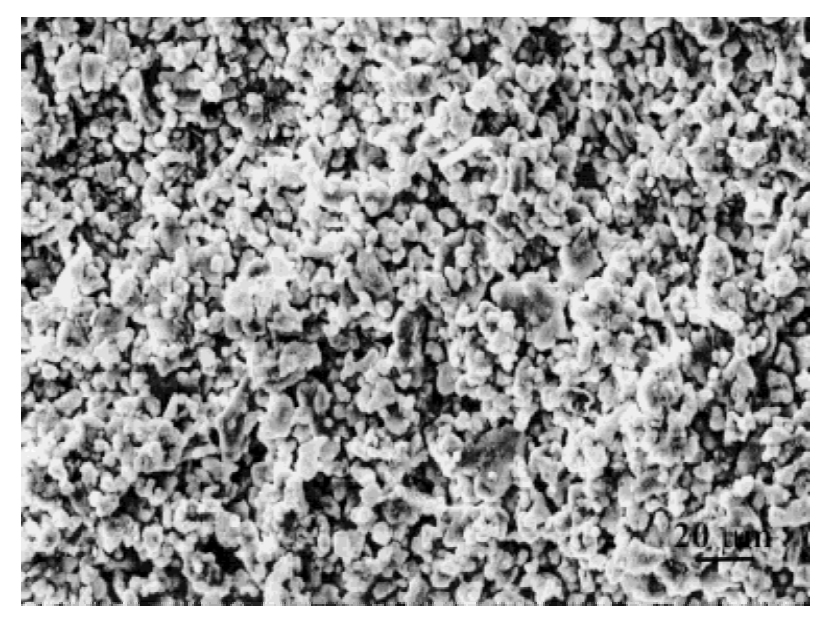

FIG. 3. The surface scanning electron microscopy shows $\alpha$-TCP cement without polyamide fibers after immersion in SBF for 10 days. 


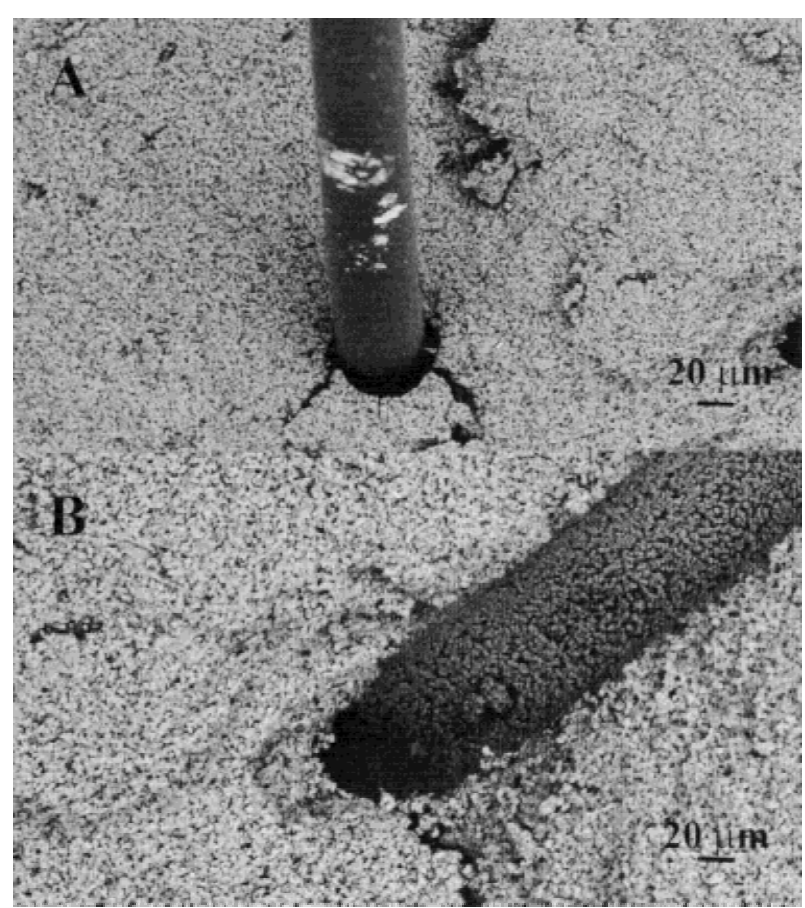

FIG. 4. The fracture scanning electron microscopy shows $\alpha$-TCP cement with polyamide fibers after immersion in SBF for 10 days.

that the fiber does not appear to have undergone plastic deformation and that it has kept its original shape. The cement shows radial fractures close to the insertion cavity of the fiber, which suggests the joining of the fiber to the matrix, as indicated by the mechanical resistance values. The fracture in the material may be acting in such as way as to increase its tenacity because part of the tensions applied to the fibers end up being transferred to the matrix and are alleviated by the appearance of radial cracks in the fibers' insertion cavity. This prevents the cracks from causing the material to collapse, owing to the distribution of tensions in the resulting cracks. The radial cracks also appear to be the cause of spacing between the matrix and the fiber, increasing the diameter of the cavity.

Figure 4B shows the cavity left by the pullout of the reinforcement fiber and the formation of a crack radial to the cavity without pullout of grains from the matrix. This demonstrates the weak coupling of the fibers, which may explain the moderate increase of compression strength. It also shows the need for surface modifiers for the fibers or additives used in the cement, such as plastifiers and deflocculants, to achieve adequate joining and the resulting increase of the mechanical resistance to higher values. Superficial treatments should involve the change in the characteristics of the polymer surface from hydrophobic to hydrophilic (15).

\section{CONCLUSIONS}

A composition of calcium phosphate cement based on $\alpha$-TCP with the addition of polyamide fibers was obtained and evaluated with the purpose of increasing the mechanical resistance. The following factors were observed. The use of polyamide fibers allowed a moderated increase in compression strength in some compositions. Higher increases of compression strength compared to those obtained in the values reported in the literature for Portland cement can be obtained only through the superficial modification of the fibers or through additives in the cement matrix that allow for improved fiber/matrix coupling as well as resulting in homogeneous fiber distribution in the cement. The variability of the measured values of mechanical resistance for the additions of polyamide fibers may be connected to nonhomogeneous distribution of fibers in the cement matrix, demonstrating its fundamental relevance in obtaining high values of mechanical resistance. And the reinforcement mechanism observed for the compositions occurred through the joining of the fiber to the matrix and the appearance of cracks radial to the insertion cavity of the fibers.

Acknowledgments: We acknowledge Project CYTED VIII.6 "Obtención y Caracterización de Materiales Compuestos con Carga de Hidroxiapatito," to which this work is connected, and the support of FAPESP (Foundation for Research Support of the São Paulo State, Brazil), Grants 98/11691-2 and 98/00563-3.

\section{REFERENCES}

1. Gruninger SE, Siew C, Chow LC, O'Young A, Ts'ao NK, Brown WE. Evaluation of the biocompatibility of a new calcium phosphate setting cement. J Dent Res 1984;63:63-200.

2. Yu D, Wong J, Matsuda Y, Fox JL, Higuchi WI, Otsuka M. Self-setting hydroxyapatite cement: A novel skeletal drug delivery system for antibiotics. J Pharm Sci 1992;81(6):529-31.

3. Driessens FCM, Fernández E, Ginebra MP, Boltong MG, Planell JA. Calcium phosphates and ceramic bone cements vs. acrylic cements. Anal Quim Int Ed 1997;93:S38-S43.

4. Monma H, Goto M, Kohmura T. Effect of additives on hydration and hardness of tricalcium phosphate. Gypsum and Lime 1984;188:11-6.

5. Bermúdez O, Boltong MG, Driessens FCM, Planell JA. Development of an octacalcium phosphate cement. J Mater Sci Mater Med 1994;5:144-6.

6. Tampieri A, Celotti G, Szontagh F, Landi E. Sintering and characterization of HA and TCP bioceramics with control of their strength and phase purity. J Mater Sci Mater Med 1997; 8:29-37.

7. Famery R, Richard N, Boch P. Preparation of $\alpha$ - and $\beta$-tricalcium phosphate ceramics with and without magnesium addition. Ceram Int 1994;20:327-36.

8. Fernández E, Ginebra MP, Boltong MG, Verbeeck RMH, Planell JA. Kinetic study of the setting reaction of a calcium phosphate bone cement. J Biomed Mater Res 1996;32:367-74. 
9. Ginebra MP, Fernández E, De Maeyer EAP, Verbeeck RMH, Boltong MG, Ginebra J, Driessens FCM, Planell JA. Setting reaction and hardening of an apatitic calcium phosphate cement. J Dent Res 1997;76(4):905-12.

10. Driessens FCM, Boltong MG, Bermudez O, Planell JA. Effective formulations for the preparation of calcium phosphate bone cements. J Mater Sci Mater Med 1994;5:164-70.

11. Fernández E, Ginebra MP, Bermudez O, Boltong MG, Driessens FCM. Dimensional and thermal behavior of calcium phosphate cements during setting compared to PMMA bone cements. J Mater Sci Lett 1995;14:4-5.
12. Beaudoin JJ. Handbook of Fiber-Reinforced Concrete. Park Ridge, NJ: Noyes Publications, 1990.

13. García Carrodeguas R, Silva Rigo E, dos Santos LA, de Oliveira LC, Boschi AO. Recubrimiento de hidroxiapatita sobre cerámica de $\mathrm{BaTiO}_{3}$. 1st Congreso Iberoamericano de Biomateriales BIOMAT'97, La Habana, May 1997.

14. Rigo ECS, Oliveira LC, Boschi AO. Efeito das Condições de Precipitação sobre as Característica Físico-Química dos Compostos do Sistema Fosfato de Cálcio. III Encontro Nacional de Biomateriais, São Paulo, Brazil. p. 9-10, May 1996.

15. Oyabu M. Japanese Patent No. 48-19,489, 1973. 